\title{
On Hall subgroups of a finite group
}

\author{
Wenbin Guo* \\ Department of Mathematics, University of Science and Technology of China, \\ Hefei 230026, P. R. China \\ E-mail: wbguo@ustc.edu.cn \\ Alexander N. Skiba ${ }^{\dagger}$ \\ Department of Mathematics, Francisk Skorina Gomel State University, \\ Gomel 246019, Belarus \\ E-mail: alexander.skiba49@gmail.com
}

November 5, 2018

\begin{abstract}
In the paper new criteria of existence and conjugacy of Hall subgroups of finite groups are given.
\end{abstract}

\section{Introduction}

Throughout this paper, all groups are finite and $G$ always denotes a finite group. We use $\mathbb{P}$ to denote the set of all primes, $\pi$ is a non-empty subset of $\mathbb{P}$ and $\pi^{\prime}=\mathbb{P} \backslash \pi$. A natural number $n$ is called a $\pi$-number if $(n, p)=1$ for any prime $p \in \pi^{\prime}$. If $|G|$ is a $\pi$-number, then $G$ is said to be a $\pi$-group.

Let $A$ and $B$ be subgroups of $G$. Then $A$ is said to permute with $B$ if $A B=B A$. If $A$ permutes with all (Sylow) subgroups of $B$, then $A$ is called quasinormal ( $S$-quasinormal, respectively) in $B$.

A group $G$ is said to be:

(a) an $E_{\pi}$-group if $G$ has a Hall $\pi$-subgroup;

(b) a $C_{\pi}$-group if $G$ is an $E_{\pi}$-group and any two Hall $\pi$-subgroups of $G$ are conjugate;

\footnotetext{
${ }^{*}$ Research of the first author is supported by a NNSF grant of China (Grant \# 11071229) and Wu Wen-Tsun Key Laboratory of Mathematics, USTC, Chinese Academy of Sciences.

${ }^{\dagger}$ Research of the second author supported by Chinese Academy of Sciences Visiting Professorship for Senior International Scientists (grant No. 2010T2J12)

Keywords: finite group, soluble group, Sylow subgroup, Hall subgroup, quasinormal subgroup, $E_{\pi}$-group, $C_{\pi}$-group, $D_{\pi}$-group.

Mathematics Subject Classification (2010): 20D20
} 
(c) a $D_{\pi}$-group if $G$ is an $E_{\pi}$-group and any $\pi$-subgroup of $G$ is contained in some Hall $\pi$-subgroup of $G$.

A group $G$ is said to be $\pi$-separable if $G$ has a chief series

$$
1=G_{0} \leq H_{1} \leq \ldots \leq H_{t-1} \leq H_{t}=G
$$

where each index $\left|H_{i}: H_{i-1}\right|$ is either a $\pi$-number or a $\pi^{\prime}$-number.

The most important result of the theory of $\pi$-separable groups is the following classical result.

Theorem A (P. Hall, S.A. C̆unihin). Any $\pi$-separable group is a $D_{\pi}$-group.

Our main goal here is to prove the following generalization of this theorem.

Theorem 1.1. Suppose that $G$ has a subgroup series

$$
1=H_{0}<H_{1} \leq \ldots \leq H_{t-1} \leq H_{t}=G
$$

where $\left|H_{i+1}: H_{i}\right|$ is either a $\pi$-number or a $\pi^{\prime}$-number for all $i=1, \ldots, t$. Suppose also that $G$ has a subgroup $T$ such that $H_{1} T=G$ and $|G: T|$ is a $\pi^{\prime}$-number. If $H_{i}$ is quasinormal in $T$, for all $i=1, \ldots, t$, then $G$ is a $D_{\pi}$-group.

The following theorem is one of the main steps in the proof of Theorem 1.1.

Theorem 1.2. Suppose that $G$ has a subgroup series

$$
1=H_{0}<H_{1} \leq \ldots \leq H_{t-1} \leq H_{t}=G
$$

where $\left|H_{i+1}: H_{i}\right|$ is either a $\pi$-number or a $\pi^{\prime}$-number for all $i=1, \ldots, t$. Suppose also that $G$ has a subgroup $T$ such that $H_{1} T=G$ and $|G: T|$ is a $\pi^{\prime}$-number. If $H_{i}$ is $S$-quasinormal in $T$, for all $i=1, \ldots, t$, then $G$ is a $C_{\pi}$-group.

Theorem 1.1 strengthens the main result in [1]. Note also that Example 1.1 in [1] shows that, under the conditions in Theorems 1.1 or 1.2 the group $G$ is not necessary $\pi$-separable.

\section{Premilaries}

Lemma 2.1. Let $N$ be a normal $C_{\pi}$-subgroup of $G$.

(i) If $G / N$ is a $C_{\pi}$-group, then $G$ is a $C_{\pi}$-group (S. A. $\breve{C}$ unihin [3]).

(ii) If $G / N$ is an $E_{\pi}$-group, then $G$ is an $E_{\pi}$-group

(iii) If $G$ has a nilpotent Hall $\pi$-subgroup, then $G$ is a $D_{\pi}$-group ([19]).

(iv) If $G$ has a Hall $\pi$-subgroup with cyclic Sylow subgroups, then $G$ is a $D_{\pi}$-group $(\mathrm{S}$. A. Rusakov [20]).

Lemma 2.2. Let $N$ be a normal $C_{\pi}$-subgroup of $G$ and $N_{\pi}$ a Hall $\pi$-subgroup of $N$. 
(i) If $G$ is a $C_{\pi}$-group, then $G / N$ is a $C_{\pi}$-group (See [4, Lemma 9]).

(ii) If every Sylow subgroup of $N_{\pi}$ is cyclic and $G / N$ is a $D_{\pi}$-group, then $G$ is a $D_{\pi}$-group (See [5] or [6, Chapter IV, Theorem 18.17]).

(iii) $G$ is a $D_{\pi}$-group if and only if $G / N$ is a $D_{\pi}$-group (See [7]).

Lemma 2.3 (O. Kegel [8]). Let $A$ and $B$ be subgroups of $G$ such that $G \neq A B$ and $A B^{x}=B^{x} A$, for all $x \in G$. Then $G$ has a proper normal subgroup $N$ such that either $A \leq N$ or $B \leq N$.

Let $A$ be a subgroup of $G$. A subgroup $T$ is said to be a minimal supplement of $A$ in $G$ if $A T=G$ but $A T_{0} \neq G$ for all proper subgroups $T_{0}$ of $G$.

The following lemma is obvious.

Lemma 2.4. If $N$ is normal in $G$ and $T$ is a minimal supplement of $N$ in $G$, then $N \cap T \leq \Phi(T)$.

Lemma 2.5 (P. Hall [10]). Suppose that $G$ has a Hall $p^{\prime}$-subgroup for each prime $p$ dividing $|G|$. Then $G$ is soluble.

Let $A$ and $B$ be subgroups of $G$ and $\varnothing \neq X \subseteq G$. Following [9], we say that $A$ is $X$-permutable (or $A X$-permutes) with $B$ if $A B^{x}=B^{x} A$ for some $x \in X$.

The following lemma is also evident.

Lemma 2.6. Let $A, B, X$ be subgroups of $G$ and $K \unlhd G$. If $A$ is $X$-permutable with $B$, then $A K / K$ is $X K / K$-permutable with $B K / K$ in $G / K$.

Lemma 2.7 (Kegel [11]). If a subgroup $A$ of $G$ permutes with all Sylow subgroups of $G$, then $A$ is subnormal in $G$.

Lemma 2.8 (H. Wielandt [12]). If a $\pi$-subgroup $A$ of $G$ is subnormal in $G$, then $A \leq O_{\pi}(G)$.

Lemma 2.9 (V. N. Knyagina and V. S. Monakhov [17]). Let $H, K$ and $N$ be subgroups of $G$. If $H$ is a Hall subgroup of $G$, then

$$
N \cap H K=(N \cap H)(N \cap K) .
$$

Lemma 2.10. Let $U \leq B \leq G$ and $G=A B$, where the subgroup $A$ permutes with $U^{b}$ for all $b \in B$. Then $A$ permutes with $U^{x}$ for all $x \in G$.

Proof. Since $G=A B, x=a b$ for some $a \in A$ and $b \in B$. Hence

$$
A U^{x}=A U^{a b}=A a\left(U^{b}\right) a^{-1}=a\left(U^{b}\right) a^{-1} A=U^{x} A .
$$

Lemma 2.11 [13, Chapter I, Lemma 1.1.19]. Let $A, B \leq G$ and $G=A B$. Then $G_{p}=A_{p} B_{p}$ for some $G_{p} \in \operatorname{Syl}_{p}(G), A_{p} \in \operatorname{Syl}_{p}(A)$ and $B_{p} \in \operatorname{Syl}_{p}(B)$.

Lemma 2.12. Let $X$ be a normal $C_{\pi}$-subgroup of $G$. Suppose that $G$ has a subgroup series

$$
1=H_{0} \leq H_{1} \leq \ldots \leq H_{t-1} \leq H_{t}=G
$$


and a supplement $T$ of $H_{1}$ in $G$ such that $H_{i} X$-permutes with every Sylow subgroup of $T$ for all $i=1, \ldots, t$. If each index $\left|H_{i+1}: H_{i}\right|$ is either a $\pi$-number or a $\pi^{\prime}$-number, then $G$ is an $E_{\pi}$-group.

Proof. Consider the subgroup series

$$
1=H_{0} X / X \leq H_{1} X / X \leq \ldots \leq H_{t-1} X / X \leq H_{t} X / X=G / X
$$

in $G / X$. If $T \leq X$, then $G=H_{1} T=X H_{1}$. Hence $G$ is an $E_{\pi}$-group by Lemma $2.1(2)$. Now let $T \not \leq X$ and $P / X$ be a Sylow $p$-subgroup of $T X / X$. Then by Lemma 2.11 there are Sylow $p$-subgroups $T_{p}$ of $T$ and $X_{p}$ of $X$ such that $T_{p} X_{p}$ is a Sylow $p$-subgroup of $T X$ and $T_{p} X_{p} X=T_{p} X=P$. Hence by Lemma 2.6, $H_{i} X / X$ permutes with every Sylow subgroup of $T X / X$ for all $i=1, \ldots, t$. On the other hand, since

$$
\left|H_{i+1} X / X: H_{i} X / X\right|=\left|H_{i+1} X: H_{i} X\right|=\left|H_{i+1}: H_{i}\right|:\left|X \cap H_{i+1}: X \cap H_{i}\right|,
$$

every index of Series (1) is either $\pi$-number or $\pi^{\prime}$-number. Hence the assertion follows from Theorem $\mathrm{B}$ in [1].

Lemma 2.13 (see Lemma in [14, Chapter A]). Let $H, K$ and $N$ be subgroups of $G$. If $H K=K H$ and $H N=N H$, then $H\langle K, N\rangle=\langle K, N\rangle H$.

\section{Base proposition}

Proposition 3.1. Let $X$ be a normal $C_{\pi}$-subgroup of $G$ and $A$ a subgroup of $G$ such that $|G: A|$ is a $\pi$-number. Suppose that $A$ has a Hall $\pi$-subgroup $A_{0}$ such that either $A_{0}$ is nilpotent or every Sylow subgroup of $A_{0}$ is cyclic. Suppose that $A X$-permutes with every Sylow p-subgroup of $G$ for all primes $p \in \pi$ or for all primes $p \in \pi \backslash\{q\}$ for some prime $q$ dividing $|G: A|$. Then $G$ is a $C_{\pi}$-group.

Proof. Assume that this proposition is false and let $G$ be a counterexample of minimal order. Then $|\pi \cap \pi(G)|>1$.

(1) $G / R$ is a $C_{\pi}$-group for any non-identity normal subgroup $R$ of $G$.

In order to prove this assertion, in view of the choice of $G$, it is enough to show that the hypothesis is still true for $(G / R, A R / R, X R / R)$. First note that $|G / R: A R / R|=|G: A R|$ is a $\pi$-number, and $A_{0} R / R$ is a Hall $\pi$-subgroup of $A R / R$ since

$$
\left|A R / R: A_{0} R / R\right|=\left|A R: A_{0} R\right|=\left|A: A \cap A_{0} R\right|=\left|A: A_{0}(A \cap R)\right| .
$$

On the other hand, $X R / R \simeq X / X \cap R$ is a $C_{\pi}$-group by Lemma 2.2 (i), and either $A_{0} R / R \simeq A_{0} / R \cap$ $A_{0}$ is nilpotent or every Sylow subgroup of $A_{0} R / R$ is cyclic. Finally, let $P / R$ be a Sylow $p$-subgroup of $G / R$, where $p \in \pi \backslash q$. Then for some Sylow $p$-subgroup $G_{p}$ we have $G_{p} R / R=P / R$. Hence $A R / R$ $X R / R$-permutes with $P / R$ by Lemma 2.6. Therefore the hypothesis holds for $(G / R, A R / R, X R / R)$.

(2) $X=1$. 
Indeed, if $X \neq 1$, then $G / X$ is a $C_{\pi}$-group by (1). Hence $G$ is $C_{\pi}$-group by Lemma 2.1 (i), a contradiction.

(3) $G$ has a proper non-identity normal subgroup $N$.

Let $p \in \pi \cap \pi(G)$, where $p \neq q$. Let $P$ be a Sylow $p$-subgroup of $G$. First assume that $A P=G$. Since $|\pi \cap \pi(G)|>1$, there is a prime $r \in \pi \cap \pi(G)$ such that $r \neq p$, so $r$ does not divide $|G: A|$. Let $R$ be a Sylow $r$-subgroup of $G$. Then for any $x \in G$ we have $A R^{x}=R^{x} A$. Hence $R \leq A_{G}$. Since $G$ is not a $C_{\pi^{-}}$group, $A \neq G$ by Lemma 2.1 (iii)(iv). Hence $1 \neq A_{G} \neq G$. Now suppose that $A P \neq G$. By (2), $P^{x} A=A P^{x}$ for all $x \in G$. Hence we have (3) by Lemma 2.3 .

(4) $N$ is a $C_{\pi}$-group.

In view of the choice of $G$ it is enough to prove that the hypothesis holds for $\left(N, A_{1}\right)$, where $A_{1}=A \cap N$. Since $|A N: A|=|N: A \cap N|,\left|N: A_{1}\right|$ is a $\pi$-number. On the other hand, $A_{0} \cap N$ is a Hall $\pi$-subgroup of $N$ since

$$
\left|A \cap N: A_{0} \cap N\right|=\left|A_{0}(A \cap N): A_{0}\right| .
$$

It clear also that either $A_{0} \cap N$ is nilpotent or every Sylow subgroup of $A_{0} \cap N$ is cyclic. Now let $N_{r}$ be any Sylow $r$-subgroup of $N$, where $r \in \pi \backslash\{q\}$. Then for some Sylow $r$-subgroup $G_{r}$ of $G$ we have $N_{r}=G_{r} \cap N$ and

$$
N \cap G_{r}=(A \cap N)\left(N \cap G_{r}\right)=A_{1} N_{r}=N_{r} A_{1}
$$

by Lemma 2.9. Therefore the hypothesis holds for $\left(N, A_{1}\right)$.

Finally, in view of (1) and (4), $G$ is a $C_{\pi}$-group by Lemma 2.1 (i), which contradicts the choice of $G$.

Corollary 3.2. Let $X$ be a normal $C_{\pi}$-subgroup of $G$ and $A$ a subgroup of $G$ such that $|G: A|$ is a $\pi$-number. Suppose that $A$ has a Hall $\pi$-subgroup $A_{0}$ such that either $A_{0}$ is nilpotent or every Sylow subgroup of $A_{0}$ is cyclic. Suppose that $G$ has a subgroup $T$ such that $|G: T|$ is a $\pi^{\prime}$-number, $G=A T$ and $A X$-permutes with every Sylow $p$-subgroup of $T$ for all primes $p \in \pi(G) \backslash \pi, p \neq q \in \mathbb{P}$. Then $G$ is a $C_{\pi^{\prime}}$-group.

Proof. Let $p \in \pi(G) \backslash \pi, p \neq q$ and $P$ be a Sylow $p$-subgroup of $G$. Since $G=A T$ and $|G: T|$ is a $\pi^{\prime}$-number, every Sylow $p$-subgroup $T_{p}$ of $T$ is a Sylow $p$-subgroup of $G$. Hence for some $x \in G$ we have $T_{p}=P^{x}$, so $A$ permutes with $P$ by Lemma 2.10. Therefore Corollary 3.2 follows from Proposition 3.1.

Corollary 3.3 ( see Theorem 1.2 in [9]). Let $X$ be a normal nilpotent subgroup of $G$ and $A$ a Hall $\pi$-subgroup of $G$. Suppose $G=A T$ and $A X$-permutes with every subgroup of $T$ Then $G$ is a $C_{\pi^{\prime}-\text { group. }}$

Corollary 3.4 (See Theorem B in [16]). Let $A$ be a Hall subgroup of a group $G$ and $T$ a minimal supplement of $A$ in $G$. Suppose that $A$ permutes with all Sylow subgroups of $T$ and with all maximal subgroups of any Sylow subgroup of $T$. Then $G$ is a $C_{\pi^{\prime}}$-group. 
Corollary 3.5 (see Theorem A* in [1]). Let $H$ be a Hall $\pi$-subgroup of $G$. Let $G=H T$ for some subgroup $T$ of $G$, and $q$ a prime. If $H$ permutes with every Sylow $p$-subgroup of $T$ for all primes $p \neq q$, then $T$ contains a complement of $H$ in $G$ and any two complements of $H$ in $G$ are conjugate.

Proof. It is clear that $T \cap H$ is a Hall $\pi$-subgroup of $T$. Moreover, if $P$ is a Sylow subgroup of $T$ and $H P=P H$, then $H P \cap T=P(H \cap T)=(H \cap T) P$. Hence by Proposition 3.1, $T$ is a $C_{\pi}$-group. Now Corollary 3.5 follows from Proposition 3.1.

Corollary 3.6 (see [18]). Let $A$ be a Hall $\pi$-subgroup of $G, G=A T$ and $A$ permutes with every subgroup of $T$. Then $G$ is an $E_{\pi^{\prime}}$-group.

\section{Proof of Theorem 1.1}

Theorem 1.2 is a special case (when $X=1$ ) of the following theorem.

Theorem 4.1. Let $X$ be a normal $C_{\pi}$-subgroup of $G$. Suppose that $G$ has a subgroup series

$$
1=H_{0}<H_{1} \leq \ldots \leq H_{t-1} \leq H_{t}=G
$$

where $\left|H_{i+1}: H_{i}\right|$ is either a $\pi$-number or a $\pi^{\prime}$-number for all $i=1, \ldots, t$. Suppose that $G$ has a subgroup $T$ such that $H_{1} T=G$ and $|G: T|$ is a $\pi^{\prime}$-number. If $H_{i}$ is $X$-permutable with each Sylow subgroup of $T$, for all $i=1, \ldots, t$, then $G$ is a $C_{\pi}$-group.

Proof. Suppose that this theorem is false and let $G$ be a counterexample of minimal order. By Lemma 2.12, $G$ has a Hall $\pi$-subgroup $S$. Hence some Hall $\pi$-subgroup $S_{1}$ of $G$ is not conjugated with $S$. Without loss of generality, we may assume that $H_{1} \neq 1$. Since $|G: T|$ is $\pi^{\prime}$-number, every Sylow $p$-subgroup $P$ of $T$, where $p \in \pi$, is also a Sylow $p$-subgroup of $G$. We proceed the proof via the following steps.

(1) $G / N$ is a $C_{\pi}$-group for every non-trivial quotient $G / N$ of $G$.

We consider the subgroup series

$$
1=H_{0} N / N \leq H_{1} N / N \leq \ldots \leq H_{t-1} N / N \leq H_{t} N / N=G / N
$$

in $G / N$. Then $\left(H_{1} N / N\right)(T N / N)=G / N$ and $|G / N: T N / N|=|G: T N|$ is a $\pi^{\prime}$-number. Moreover, by Lemma 2.6, $H_{i} N / N$ is $X N / N$-permutable with every Sylow subgroup of $T N / N$ for all $i=1, \ldots, t$. On the other hand, since

$$
\left|H_{i+1} N / N: H_{i} N / N\right|=\left|H_{i+1} N: H_{i} N\right|=\left|H_{i+1}: H_{i}\right|:\left|N \cap H_{i+1}: N \cap H_{i}\right|,
$$

every index of the series (2) is either $\pi$-number or $\pi^{\prime}$-number. Moreover, $X N / N \simeq X /(X \cap N)$ is a $C_{\pi}$-group by Lemma 2.2 (i). All these show that the hypothesis holds for $\mathrm{n} G / N$. Hence in the case, where $N \neq 1, G / N$ is a $C_{\pi}$-group by the choice of $G$.

(2) $O_{\pi^{\prime}}(G)=1=O_{\pi}(G)$. 
Suppose that $D=O_{\pi^{\prime}}(G) \neq 1$. Then by (1), there is an element $x \in G$ such that $S_{1}{ }^{x} D=S D$. But by the Schur-Zassenhaus theorem, $S_{1}{ }^{x}$ and $S$ are conjugate in $S D$, which implies that $S_{1}$ and $S$ are conjugate in $G$. This contradiction shows that $O_{\pi^{\prime}}(G)=1$. Analogously, one can prove that $O_{\pi}(G)=1$.

(3) $X=1$ (This follows from (1), Lemma 2.1 (i) and the choice of $G$ ).

(4) $T \neq G$.

Suppose that $T=G$. Then by hypothesis and (3), $H_{1}$ permutes with all Sylow subgroups of $G$. It follows from Lemma 2.7 that $H_{1}$ is subnormal in $G$. Since $H_{1}$ is either a $\pi$-group or a $\pi^{\prime}$-group, $H_{1} \leq O_{\pi}(G)$ or $H_{1} \leq O_{\pi^{\prime}}(G)$ by Lemma 2.8. It follows from (2) that $H_{1}=1$, which contradicts to our assumption about $H_{1}$. Hence (4) holds.

(5) The hypothesis holds for $T$.

Consider the subgroup series

$$
1=H_{0} \cap T \leq H_{1} \cap T \leq \ldots \leq H_{t-1} \cap T \leq H_{t} \cap T=T
$$

of the group T. Since

$$
H_{i+1}=H_{i} T \cap H_{i+1}=H_{i}\left(H_{i+1} \cap T\right)
$$

we have

$$
\left|H_{i+1}: H_{i}\right|=\left|H_{i+1} \cap T: H_{i} \cap T\right|,
$$

for all $i=1, \ldots, t-1$ and $\left|H_{1} \cap T: H_{0} \cap T\right|=\left|H_{1} \cap T\right|$ divides $\left|H_{1}: 1\right|$, we see that every index of the series (3) is either $\pi$-number or $\pi^{\prime}$-number. Now let $E$ be a Sylow subgroup of $T$. By (3) and the hypothesis, $H_{i} E=E H_{i}$. Hence

$$
H_{i} E \cap T=E\left(H_{i} \cap T\right)=\left(H_{i} \cap T\right) E .
$$

This shows that the hypothesis holds for $T$.

(6) $T$ is a $C_{\pi}$-group.

Since $T \neq G$ by (4), and the hypothesis holds for $T$ by (5), the minimal choice of $G$ implies that (6) holds.

(7) $T$ is a $E_{\pi^{\prime} \text {-Subgroup. }}$

This follows from (3), (5) and Lemma 2.12.

(8) Let $T_{\pi^{\prime}}$ be a Hall $\pi^{\prime}$-subgroup of $T$ and $D$ a normal subgroup of $G$. Then $T_{\pi^{\prime}} \neq 1$, and if either $H_{1} \leq D$ or $T_{\pi^{\prime}} \leq D$, then $D=G$.

Suppose that $T_{\pi^{\prime}}=1$. Then $H_{1}$ is a Hall $\pi^{\prime}$-subgroup of $G$. Therefore $G$ is a $C_{\pi^{-}}$-group by Corollary 3.2, a contradiction. Hence $T_{\pi^{\prime}} \neq 1$.

We show that the hypothesis holds for $D$. Consider the subgroup series

$$
1=D_{0} \leq D_{1} \leq \ldots \leq D_{t-1} \leq D_{t}=D,
$$


where $D_{i}=H_{i} \cap D$ for all $i=1, \ldots, t$. Let $T_{0}=D \cap T$. First we show that $D_{1} T_{0}=D$. If $H_{1} \leq D$, then

$$
D=H_{1}(D \cap T)=H_{1} T_{0}=D_{1} T_{0} .
$$

Now suppose that $T_{\pi^{\prime}} \leq D$. In view of (3), $H_{1}$ permutes with $T_{\pi^{\prime}}$. Since $H_{1} T=G, T \neq G$ and $|G: T|$ is a $\pi^{\prime}$-number, $H_{1}$ is a $\pi^{\prime}$-group. Hence $H_{1} T_{\pi^{\prime}}$ is a Hall $\pi^{\prime}$-subgroup of $G$. Therefore

$D=\left(D \cap H_{1} T_{\pi^{\prime}}\right)\left(D \cap T_{\pi}\right)=T_{\pi^{\prime}}\left(D \cap H_{1}\right)\left(D \cap T_{\pi}\right)=\left(D \cap H_{1}\right)\left(T_{\pi^{\prime}}\left(D \cap T_{\pi}\right)\right)=\left(D \cap H_{1}\right)(D \cap T)=D_{1} T_{0}$.

It is also clear that $\left|D: T_{0}\right|$ is a $\pi^{\prime}$-number. Now let $P$ be a Sylow $p$-subgroup of $T_{0}$. Then for some Sylow $p$-subgroup $T_{p}$ of $T$ we have $P=T_{p} \cap D$. Hence in view of Lemma 2.9,

$$
D \cap H_{i} T_{p}=\left(D \cap H_{i}\right)\left(D \cap T_{p}\right)=D_{i} P=P D_{i} .
$$

Thus for any $p \in \pi, D_{i}$ is permutable with every Sylow $p$-subgroup of $T_{0}$ for all $i=1, \ldots, t$. Finally, since the number

$$
\left|D_{i}: D_{i-1}\right|=\left|\left(D \cap H_{i}\right) H_{i-1}: H_{i-1}\right|
$$

divides $\left|H_{i}: H_{i-1}\right|$, each index $\left|D_{i}: D_{i-1}\right|$ is either a $\pi$-number or a $\pi^{\prime}$-number. Therefore the hypothesis holds for $D$. Suppose that $D \neq G$. Then $D$ is a $C_{\pi}$-group by the choice of $G$. Since either $1 \neq H_{1} \leq D$ or $1 \neq T_{\pi^{\prime}} \leq D, G / D$ is a $C_{\pi^{-}}$group by (1). It follows from Lemma 2.1 (i) that $G$ is a $C_{\pi}$-group, which contradicts the choice of $G$. Hence, (8) holds.

Final contradiction. Since $G=H_{1} T$ and $H_{1}$ permutes with all Sylow subgroups of $T$ by (3),

$$
H_{1}\left(T_{\pi^{\prime}}\right)^{x}=\left(T_{\pi^{\prime}}\right)^{x} H_{1}
$$

for all $x \in G$ by Lemma 2.10. Therefore by Lemma 2.3, either $H_{1}{ }^{G} \neq G$ or $\left(T_{\pi^{\prime}}\right)^{G} \neq G$. But in view of (8) both these cases are impossible. The contradiction completes the proof of the result.

Corollary 4.2 (see Theorem 5.1 in [1]). Let $X$ be a normal $\pi$-separable subgroup of $G$. Suppose that $G$ has a subgroup series

$$
1=H_{0}<H_{1} \leq \ldots \leq H_{t-1} \leq H_{t}=G
$$

where $\left|H_{i+1}: H_{i}\right|$ is either a $\pi$-number or a $\pi^{\prime}$-number for all $i=1, \ldots, t$. Suppose that $G$ has a subgroup $T$ such that $H_{1} T=G$ and $|G: T|$ is a $\pi^{\prime}$-number. If $H_{i}$ is $X$-permutable with each subgroup of $T$, for all $i=1, \ldots, t$, then $G$ is a $C_{\pi}$-group.

Theorem 1.1 is a special case (when $X=1$ ) of the following theorem.

Theorem 4.3. Let $X$ be a normal an $E_{\pi}$-subgroup of $G$ and $X_{\pi}$ a Hall $\pi$-subgroup of $X$. Suppose that $G$ has a subgroup series

$$
1=H_{0}<H_{1} \leq \ldots \leq H_{t-1} \leq H_{t}=G,
$$

where $\left|H_{i+1}: H_{i}\right|$ is either a $\pi$-number or a $\pi^{\prime}$-number for all $i=1, \ldots, t$. Suppose that $G$ has a subgroup $T$ such that $H_{1} T=G$ and $|G: T|$ is a $\pi^{\prime}$-number. 
(i) Suppose that the Sylow subgroups of $X_{\pi}$ are cyclic. If $H_{i}$ is $X$-permutable with each cyclic subgroup $H$ of $T$ of prime power order, for all $i=1, \ldots, t$, then $G$ is a $D_{\pi}$-group.

(ii) Suppose that $X$ is a $D_{\pi}$-subgroup. If $H_{i}$ is $X$-permutable with each cyclic subgroup $H$ of $T$ of prime power order, for all $i=1, \ldots, t$, then $G$ is a $D_{\pi}$-group.

Proof. (i) Suppose that this assertion is false and let $G$ be a counterexample of minimal order. In view of Theorem 4.1, $G$ is a $C_{\pi}$-group. Hence there is a $\pi$-subgroup $U$ of $G$ such that for any Hall $\pi$-subgroup $E$ of $G$ we have $U \not \leq E$.

(1) $G / N$ is a $D_{\pi}$-group for any non-identity normal subgroup $N$ of $G$.

Consider the subgroup series

$$
1=H_{0} N / N \leq H_{1} N / N \leq \ldots \leq H_{t-1} N / N \leq H_{t} N / N=G / N
$$

It is clear that $\left(H_{1} N / N\right)(T N / N)=G / N,|G / N: T N / N|$ is a $\pi^{\prime}$-number and $\left|H_{i+1} N / N: H_{i} N / N\right|$ is either a $\pi$-number or a $\pi^{\prime}$-number for all $i=1, \ldots, t$ (see (1) in the proof of Theorem 4.1). Now let $H / N$ be a cyclic subgroup of $T N / N$ of prime power order $|H / N|$. Then $H=N(H \cap T)$. Let $W$ be a group of minimal order with the properties that $W \leq H \cap T$ and $N W=H$. If $N \cap W \not \leq \Phi(W)$, then for some maximal subgroup $S$ of $W$ we have $(N \cap W) S=W$. Hence $H=N W=N(N \cap W) S=N S$, a contradiction. Hence $N \cap W \leq \Phi(W)$. Since $W / W \cap N \simeq H / N$ is a cyclic group of prime power order, it follows that $W$ is cyclic group of prime power order. Hence $H_{i}$ is $X$-permutable with $W$. Thus $H_{i} N / N$ is $X N / N$-permutable with $W N / N=H / N$ by Lemma 2.6. Therefore the hypothesis holds for $G / N$. But since $N \neq 1,|G / N|<|G|$ and so $G / N$ is a $D_{\pi}$-group by the choice of $G$.

(2) $X=1$.

Suppose that $X \neq 1$. Then $G / X$ is a $D_{\pi}$-group by (1). Hence $G$ is a $D_{\pi}$-group by Lemma 2.2 (iii), a contradiction. Thus we have (2).

(3) $H_{1}$ permutes with every subgroup of $U$.

Let $Z$ be any cyclic subgroup of $U$ of prime power order $p^{n}$. Then $p \in \pi$ and $Z \leq G_{p}$ for some Sylow $p$-subgroup $G_{p}$ of $G$. Since $|G: T|$ is a $\pi^{\prime}$-number, there is an element $x=h t$ such that $\left(G_{p}\right)^{x} \leq T$. Hence $H_{1} Z=Z H_{1}$ by Lemma 2.10 , which in view of Lemma 2.13 implies that $H_{1} U=U H_{1}$.

(4) $T \neq G$ (see the proof of (4) in the proof of Theorem 4.1).

(5) $T$ is a $D_{\pi}$-group.

The hypothesis holds for $T$ (see (5) in the proof of Theorem 4.1), so in view of (4) the minimal choice of $G$ implies that we have (5).

(6) $V=H_{1} U$ is a $C_{\pi}$-group.

Indeed, $V$ is a group by (3), and since $T \neq G, H_{1}$ is a Hall $\pi^{\prime}$-subgroup of $V$. Therefore we have (6) by (2) and Corollary 3.2 . 
(7) $|V: T \cap V|$ is a $\pi^{\prime}$-number and $T \cap V$ is a $C_{\pi}$-group.

First note that $|G: T|=|V: T \cap V|$ is a $\pi^{\prime}$-number. But $H_{1}$ is a Hall $\pi^{\prime}$-subgroup of $V$. Hence $V=H_{1}(T \cap V)$, which implies that

$$
\left.\left|V: H_{1}\right|=\mid T \cap V: H_{1} \cap T \cap V\right) \mid
$$

is a $\pi$-number. Hence $A=H_{1} \cap T \cap V$ is a Hall $\pi^{\prime}$-subgroup of $T \cap V$. Finally, if $W$ is any $\pi$-subgroup of $T \cap V=$, then $H_{1} W=W H_{1}$ by (3). Therefore

$$
A W=\left(H_{1} \cap T \cap V\right) W=\left(H_{1} W \cap T \cap V\right)=W A .
$$

Hence $T \cap V$ is a $C_{\pi}$-group by Proposition 3.1.

Final contradiction for (i). In view of (7), $|V: T \cap V|$ is a $\pi^{\prime}$-number and $T \cap V$ is a $C_{\pi^{-}}$-group. Hence in view of (6), there is an element $x \in V$ such that $U^{x} \leq T \cap V \leq T$. But by (5), $T$ is a $D_{\pi}$-group. Hence for some Hall $\pi$-subgroup $T_{\pi}$ of $T$ we have $U \leq T_{\pi}$, which is a contradiction since $T_{\pi}$ is clearly a Hall $\pi$-subgroup of $G$.

(ii) See the proof of (i) and use Lemma 2.2 (iii).

\section{Groups with soluble Hall $\pi$-subgroups}

A group $G$ is said to be:

(a) an $E_{\pi}^{S}$-group ( $E_{\pi}^{N}$-group) if $G$ has a soluble (a nilpotent, respectively) Hall $\pi$-subgroup;

(b) a $C_{\pi}^{S}$-group $\left(C_{\pi}^{N}\right.$-group) if $G$ has a soluble (nilpotent, respectively) Hall $\pi$-subgroup and $G$ is a $C_{\pi}$-group;

(c) a $D_{\pi}^{S}$-group if $G$ is a $C_{\pi}^{S}$-group and any $\pi$-subgroup of $G$ is contained in some Hall $\pi$-subgroup of $G$.

Our next results are new criteria for a group to be an $E_{\pi}^{S}$-group.

Lemma 5.1. Let $N$ be a normal $E_{\pi}$-subgroup of $G$ and $N_{\pi}$ a Hall $\pi$-subgroup of $N$. Suppose that $N_{\pi}$ is nilpotent. If $G / N$ is a $D_{\pi}^{S}$-group, then $G$ is a $D_{\pi}^{S}$-group (See [4] or [6, Chapter IV, Theorem 18.15]).

Theorem 5.2. Let $X$ be a normal $E_{\pi}^{N}$-subroup of $G$. Suppose that $G$ has a subgroup series

$$
1=H_{0}<H_{1} \leq \ldots \leq H_{t-1} \leq H_{t}=G
$$

where $\left|H_{i+1}: H_{i}\right|$ is divisible by at most one prime in $\pi$, for all $i=1, \ldots, t$. Suppose that $G$ has a subgroup $T$ such that $H_{1} T=G$ and $|G: T|$ is a $\pi^{\prime}$-number. If $H_{i}$ is $X$-permutable with each Sylow subgroup of $T$, for all $i=1, \ldots, t$, then $G$ is an $E_{\pi}^{S}$-group.

Proof. Suppose that this theorem is false and let $G$ be a counterexample of minimal order. 
(1) Every non-trivial quotient $G / N$ of $G$ is an $E_{\pi}^{S}$-group (See (1) in the proof of Theorem 4.1).

(2) $X=1$.

Suppose that $X \neq 1$. Then $G / X$ of $G$ is an $E_{\pi}^{S}$-group by (1). Let $E / X$ be a soluble $\pi$-Hall subgroup of $G / X$. Then, by Lemma $5.1, E$ is a $D_{\pi}^{S}$-group and if $U$ is a Hall subgroup of $E$, then $U$ is a Hall subgroup of $G$. Hence $G$ is an $E_{\pi}^{S}$-group, a contradiction. Thus we have (2).

(3) $T \neq G$.

Suppose that $T=G$. Then by hypothesis and (2), $H_{1}$ permutes with all Sylow subgroups of $G$. Hence $H_{1}$ is subnormal in $G$ by Lemma 2.7 , so

$$
1<H_{1} \leq O_{\pi^{\prime} \cup\{p\}}(G)
$$

for some $p \in \pi$ by Lemma 2.8. Therefore $O_{\pi^{\prime} \cup\{p\}}(G)$ is a non-identity normal $D_{\pi}^{N}$-group-subgroup of $G$, which as in the proof of (2), conducts us to the contradiction. Thus (3) holds.

Final contradiction. In view of (2), the hypothesis is true for $T$ (see (5) in the proof of Theorem 4.1). Hence $T$ is an $E_{\pi}^{S}$-group by (3) and the choice of $G$. But since $|G: T|$ is a $\pi^{\prime}$-number, any Hall $\pi$-subgroup of $T$ is a Hall $\pi$-subgroup of $G$ as well. Hence $G$ is an $E_{\pi}^{S}$-group, a contradiction.

The theorem is proved.

Theorem 5.3. Let $X$ be a normal $E_{\pi}^{N}$-subgroup of $G$. Suppose that $G$ has a subgroup series

$$
1=H_{0}<H_{1} \leq \ldots \leq H_{t-1} \leq H_{t}=G
$$

where $\left|H_{i+1}: H_{i}\right|$ is divisible by at most one prime in $\pi$, for all $i=1, \ldots, t$. Suppose that $G$ has a subgroup $T$ such that $H_{1} T=G$ and $|G: T|$ is a $\pi^{\prime}$-number. If $H_{i}$ is $X$-permutable with each cyclic subgroup $H$ of $T$ of prime power order, for all $i=1, \ldots, t$, then $G$ is a $D_{\pi}^{S}$-group.

Proof. Suppose that this theorem is false and let $G$ be a counterexample of minimal order. Then $|\pi \cap \pi(G)|>1$. Without loss we may suppose that $H_{1} \neq 1$ and $H_{t-1} \neq G$.

(1) Every on-trivial quotient $G / N$ of $G$ is a $D_{\pi}^{S}$-group (See (1) in the proof of Theorem 4.3).

(2) If $N$ is a normal $E_{\pi}^{N}$-subgroup of $G$, then $N=1$. In particular, $X=1$.

Suppose that $N \neq 1$ Then $G / N$ of $G$ is a $D_{\pi}^{S}$-group by (1) and the choice of $G$. Hence $G$ is a $D_{\pi}^{S}$-group by Lemma 5.1, which contradicts the choice of $G$. Hence we have (2).

(3) $T \neq G$ (See (3) in the proof of Theorem 5.2).

(4) The hypothesis holds for $H_{t-1}$ and $T$.

Since

$$
H_{t-1}=H_{1}\left(H_{t-1} \cap T\right)
$$

and

$$
\left|H_{t-1}: H_{t-1} \cap T\right|=|G: T|,
$$


the hypothesis holds for $H_{t-1}$ by (2). The second assertion of (4) may be proved as (5) in the proof of Theorem 4.1.

(5) $G$ is a $C_{\pi}^{S}$-group.

Since $\left|G: H_{t-1}\right|$ is divisible by at most one prime in $\pi$ and $|\pi \cap \pi(G)|>1$, there is a prime $p \in \pi \cap \pi(G)$ such that for a Sylow $p$-subgroup $P$ of $G$ we have $P \leq H_{t-1}$, so in view of (2) and Lemma 2.10 we have $1<P \leq\left(H_{t-1}\right)_{G}$. Since $H_{t-1} \neq G$ and the hypothesis holds for $H_{t-1}, H_{t-1}$ is a $D_{\pi}^{S}$-group by the choice of $G$. Hence $\left(H_{t-1}\right)_{G}$ is a $C_{\pi}^{S}$-group, so $G$ is a $C_{\pi}^{S}$-group by Lemma 2.1 (i) and Theorem 5.2.

(6) For any $i, H_{i}$ permutes with every $\pi$-subgroup of $G$ (see (3) in the proof of Theorem 4.3).

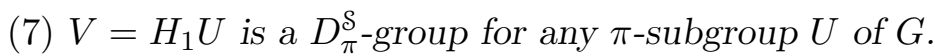

By (6), $V$ is a subgroup of $G$. Moreover, $V$ is a $C_{\pi}$-group by Proposition 3.1 . We show that the hypothesis holds for $V$. Let $V_{\pi}$ be a Hall $\pi$-subgroup of $V$. Then $V=H_{1} V_{\pi}$ and

$$
V_{i}=V \cap H_{i}=H_{1}\left(V_{\pi} \cap H_{i}\right),
$$

for all $i=1, \ldots, t$. Let $W$ be any subgroup of $V_{\pi}$. Then

$$
V_{i} W=\left(H_{1}\left(V_{\pi} \cap H_{i}\right)\right) W=H_{1}\left(V_{\pi} \cap H_{i} W\right)=H_{1} W\left(V_{\pi} \cap H_{i}\right) W\left(H_{1}\left(V_{\pi} \cap H_{i}\right)\right)=W V_{i} .
$$

It is clear also that $\left|V_{i+1}: V_{i}\right|$ is divisible by at most one prime in $\pi$, for all $i=0, \ldots, t$. Hence the hypothesis holds for $V$. Suppose that $G=V$. In this case, in view of (5), we may suppose that $V_{\pi}$ is a soluble Hall $\pi$-subgroup of $G$. Let $L$ be a minimal normal subgroup of $V_{\pi}$. Then

$$
L^{G}=L^{V_{\pi} H_{1}}=L^{H_{1}} \leq L H_{1} \cap L^{H_{1}}=L\left(L^{G} \cap H_{1}\right)
$$

and $L$ is a $q$-group for some $q \in \pi$. From (2) it follows that for some prime $p \in \pi$ with $q \neq p$ we have $p \in \pi\left(L^{G} \cap H_{1}\right)$ and a Sylow $p$-subgroup $P$ of $L^{G} \cap H_{1}$ is also a Sylow subgroup of $L^{G}$. For some Sylow $p$-subgroup $G_{p}$ of $G$ we have $P=L^{G} \cap G_{p}$, so

$$
L^{G} \cap H_{1} G_{p}=\left(L^{G} \cap H_{1}\right)\left(L^{G} \cap G_{p}\right)=\left(L^{G} \cap H_{1}\right) P=P\left(L^{G} \cap H_{1}\right) .
$$

Hence $P \leq\left(L^{G} \cap H_{1}\right)_{L^{G}}$. Therefore $G$ has a non-identity subnormal subgroup $R=\left(L^{G} \cap H_{1}\right)_{L^{G}}$ of order divisible by at most one prime in $\pi$, which contradicts (2). Hence $V \neq G$, so $V$ is a $D_{\pi}^{S}$-group by the choice of $G$.

Final contradiction. Let $U$ be any $\pi$-subgroup of $G$ and $V=H_{1} U$. Then $V$ is a $D_{\pi}^{S}$-subgroup of $G$ by (7), and $|G: T|=|V: V \cap T|$ is a $\pi^{\prime}$-number. By (3) and (4), $T$ is a $D_{\pi^{-} \text {-group. Hence for }}$ some Hall $\pi$-subgroup $V_{\pi}$ of $V$ we have $U \leq V_{\pi}$, and $V_{\pi} \leq T_{\pi}$. But since $|G: T|$ is a $\pi^{\prime}$-number, $T_{\pi}$ is a Hall $\pi$-subgroup of $G$. Therefore $G$ is a $D_{\pi}^{S}$-group.

Corollary 5.4. Suppose that $G$ has a subgroup series

$$
1=H_{0}<H_{1} \leq \ldots \leq H_{t-1} \leq H_{t}=G,
$$


where $\left|H_{i+1}: H_{i}\right|$ is divisible by at most one prime in $\pi$, for all $i=1, \ldots t$. Suppose also that $G$ has a subgroup $T$ such that $H_{1} T=G$ and $|G: T|$ is a $\pi^{\prime}$-number. If $H_{i}$ is quasinormal in $T$, for all $i=1, \ldots, t$, then $G$ is a $D_{\pi}^{S}$-group.

Corollary 5.6 (see S. A. C̆unihin [21] or [6, Chapter IV, Theorem 18.13]). If $G$ has a chief subgroup series

$$
1=H_{0}<H_{1} \leq \ldots \leq H_{t-1} \leq H_{t}=G,
$$

where $\left|H_{i+1}: H_{i}\right|$ is divisible by at most one prime in $\pi$, for all $i=1, \ldots, t$, then $G$ is a $D_{\pi}^{S}$-group.

\section{References}

[1] W. Guo, A.N. Skiba, New criterions of existence and conjugacy of Hall subgroups of finite groups, Proc. Amer. Math. Soc., 2011, 139, 2327-2336.

[2] S. A. $\breve{C}$ unihin, On weakening the conditions in theorems of Sylow's type, Doklady Akad. Nauk SSSR (NS), 83 (1952), 663-665.

[3] S. A. Cunihin, On existence and conjugacy of subgroups of a finite group, Mat. Sbornik, , 1953, 33 (1), 111-132.

[4] P. Hall, Theorems like Sylow's, Proc. London Math. Soc., 1956, 6 (3), 286-304.

[5] L.A. Shemetkov, On Sylow properties of finite groups, DAN BSSR, 1972, 16 (10), 881-883.

[6] L.A. Shemetkov, Formations of Finite Groups, Nauka, Main Editorial Board for Physical and Mathematical Literature, Moscow, 1978.

[7] D.O. Revin, E.P. Vdovin, Hall subgroups of finite groups, Contemp. Math., 2006, 402, 229-265.

[8] O. H. Kegel, Produkte nilpotenter Gruppen, Arch. Math., 1961, 12, 90-93.

[9] Wenbin Guo, K. P. Shum and Alexander N. Skiba, $X$-semipermutable subgroups of finite groups, J. Algebra, 2007, 315, 31-41.

[10] P. Hall, A characteristic property of soluble groups, J. London Math. Soc., 1937, 12, 188-200.

[11] O. Kegel, Sylow-Gruppen and Subnormalteiler endlicher Gruppen, Math. Z., 1962, 78 205-221.

[12] H. Wielandt, Subnormal subgroups and permutation groups. Lectures given at the Ohio State University, Columbus, Ohio, 1971.

[13] A. Ballester-Bolinches, R. Esteban-Romero, M. Asaad, Products of Finite Groups, Walter de Gruyter, Berlin, New York, 2010.

[14] K. Doerk, T. Hawkes, Finite Soluble Groups, Walter de Gruyter, Berlin, New York, 1992. 
[15] Wenbin Guo, K.P. Shum and Alexander N. Skiba, Schur-Zassenhaus Theorem for $X$-permutable subgroups, Algebra Colloquium, 2008, 15(2), 185-192.

[16] W. Guo and A.N. Skiba, Criterions of Existence of Hall Subgroups in Non-soluble Finite Groups, Acta Math. Sinica, 2010, 26(2), 295-304.

[17] B.N. Knyagina, V.S. Monakhov, On $\pi^{\prime}$-properties of finite group having a Hall $\pi$-subgroup, Siberian. Math. J., 2011, 52 (2), 298-309.

[18] N. Foguel, On seminormal subgroups, J. Algebra, 1994, 165, 633-635.

[19] H. Wielandt, Zum Satz von Sylow, II., Math. Z., 1959, 71, 461-462.

[20] S.A. Rusakov, Analogous of theorem of Sylow on existence and embedding subgroups, Siberian Math. J., 1963, 4(5), 325-342.

[21] S. A. C̆unihin, On $\pi$-separate groups, Doklady Akad. Nauk. SSSR, 1948, 59, 443-445. 